\title{
Magnitude of Elective Surgical Patient Cancelation and Associated Factors at Assella Teaching and Referral Hospital, Oromia Region, South East Ethiopia
}

\author{
Abdurahman Tune Dedecho ${ }^{*}$, Beleaneh Tefera Geda, Gemechis Kabe Gonfa \\ Department of Anesthesia, Arsi University, Asella, Ethiopia \\ Email address: \\ abdurahmantune@gmail.com (A. T. Dedecho) \\ ${ }^{*}$ Corresponding author

\section{To cite this article:} \\ Abdurahman Tune Dedecho, Beleaneh Tefera Geda, Gemechis Kabe Gonfa. Magnitude of Elective Surgical Patient Cancelation and \\ Associated Factors at Assella Teaching and Referral Hospital, Oromia Region, South East Ethiopia. Clinical Medicine Research. \\ Vol. 9, No. 4, 2020, pp. 91-96. doi: 10.11648/j.cmr.20200904.14
}

Received: June 17, 2020; Accepted: July 21, 2020; Published: August 10, 2020

\begin{abstract}
Abstarct: Back ground: Elective surgery is a term used for non-emergency surgery which is medically Necessary, but which can be delayed for at least 24 hours. Elective surgery is usually performed in an operating theatre or procedure room under some form of Anesthesia by a surgeon. Operation theatre requires considerable human resources and expenditure from Hospital budget. Objective: To assess the magnitude of cancelation of elective operations and associated factors among elective surgical patients at Arsi University, Assella Referral and Teaching Hospital. Method: a cross sectional hospital based study was conducted at Arsi University, Assella Referral and Teaching Hospital from April 20 to June 1, 2018. The total sample size was 112 elective surgical patients Who was planned to have a surgery at ATRH. The data was collected by reviewing the daily schedule lists for elective surgery with a predesigned form. The data was analyzed using SPSS software version 20. Result: a total of 112 patients were planned to be operated during our study period; from those $36(32.2 \%)$ was canceled. Rate of cancelation in male and female is $36.6 \%, 27 \%$ respectively. surgery department has the highest number of cancelation procedures $19(52.7 \%)$ The most common reasons for cancellations were administration related from surgical side and shortage of time 17 (47.2\%), medical reasons $9(25 \%)$ and inadequate preparation of the patient 7 (16.6\%). Conclusion: Cancellation of elective surgical procedures on the scheduled day of surgery was high during the study. Most of the reasons were shortage of time and management related, medical reasons and inadequate preparation of patient. Most of the reasons were avoidable.
\end{abstract}

Keywords: Cancellation, Surgery, Panned Procedure

\section{Background}

Elective surgery is a term used for non-emergency surgery which is medically necessary, but which can be delayed for at least 24 hours. Patients requiring emergency surgery will not be placed on the elective surgery list. Elective surgery is usually performed in an operating theatre or procedure room under some form of anesthesia by a surgeon [1].

Cancellation of elective planned surgical case is a known quality problem in healthcare system that harms patients and wastes resources, leading to increase healthcare costs. Reasons for cancellations are complex because they are related to patients, organizational issues, and clinical staff [2].
Increasing patient satisfaction through efficient practice is an appropriate objective of a healthcare system. A high cancellation rate for elective surgical procedures makes it difficult to accomplish this. Cancellation reduces operating room efficiency and increases costs $[3,4]$. Cancellation of operations in hospitals is a significant problem with far reaching consequences [5].

However, different definitions of cancellation exist in the international literature. Some authors define 'cancellation' as only those procedures that were cancelled on the day on which surgery was scheduled, whereas others also include 
those that were cancelled on the previous day.

Operation theatre is the heart of a hospital requiring considerable human resources and expenditure from hospital budget. However, Operation theatres are Underutilized and lie idle most of the time and many patients who are called for Operations from waiting list are not operated upon [4, 5].

Clearly, the cost-effective use of surgical facilities necessitates efficient use of Theatre time and personnel. Repeated delays and cancellations result in increased costs for hospitals and frustration and anxiety for patients [3, 7]. Cancellation of surgical operations in hospitals is a significant problem with many undesirable consequences. Cancelled operations can annoy patients and their Families. They are a major drain on health resources, increases theatre costs, results in wasted operating room time and decreases efficiency. In spite of the extensive available literature on preparation of surgical patients and performance of surgical procedures, the focus given to the cancellation of planned surgical Operation has been quiet restricted globally [8, 9].

Appreciation of the usual reasons for cancellations can improve theatre utilization by permitting administrators and clinicians to anticipate those cases in which Problems might arise so that additional attention can be paid to them. The Cancellation of surgery creates hardship for patients, who often plan their working and family lives around the proposed operation date. Most are cancelled at less than 24 hours' notice. The cost implications to the patients, their families and the Institutions must be significant but have not been calculated [7].

These problems are again influenced by demographic, poor socio-economic status and poor quality of services, cancellation rates ranging between $19 \%$ and $33 \%$ were reported from developing countries. University hospitals had significantly higher cancellation rates than did smaller hospitals, and among the services, general surgery services had the highest cancellation rates $[6,8]$.

The incidence of cancellation of elective surgical procedures on the scheduled day of surgery is high in Jimma. Most of the causes of the cancellations are potentially preventable [9].

Despite there are researches similar to this title worldwide their results are to different both as a prevalence and associated factors, the overall cancelation rate was $6.5 \%$ in Spanish General Hospital but it is $23.0 \%$ in research done in Jimma Ethiopia [9, 10].

Patients who are planned to undergo elective surgery in Assella Teaching and Referral Hospital faces the problem of cancelation most of the time based on revision of recordings. As such this has to be looked into to find out what are factors associated with cancelation of elective surgery. Most of the reasons of postponement of elective general surgical operations were avoidable in previous researches and can be prevented by simple steps so the problem is on finding out those problems. The assessment of cancelation and associated factors identify prevalence of the problem and major factors leads to cancelation, for policy makers in ATRH, researchers and ultimately to prevent cancelation.

\section{Methods and Materials}

\subsection{Study Area}

The study was conducted at Assella Teaching and Referral Hospital which is found in Assella town. Assella is a town and separate woreda in South East Ethiopia, which located in the Arsi Zone of the Oromia Region about 175 kilometers from Addis Ababa, this city has a latitude and longitude of $7^{\circ} 57^{\prime} \mathrm{N} 39^{\circ} 7^{\prime} \mathrm{E}$, with an elevation of 2,430 meters. There are 10 Anesthetists currently working in Assella Referral and Teaching HospitalThe hospital gives service of surgery in different departments and has four functional tables:

Surgery Gynecology and Obstetrics Pediatrics and ophthalmology surgery

\subsection{Study Design and Period}

A hospital based cross sectional study was done from A mril to June 2010 E. C.

Source Population: all surgical cases (both elective and emergency cases) that undergo surgery in ATRH.

Study population: All patients scheduled for different elective surgical procedures in ATRH during the study period.

Inclusion criteria

All patients scheduled for different elective surgical procedures in ATRH during the study period were included.

Exclusion criteria

listed for elective surgery but were done before the day of schedule as emergency.

Sample Size and Sampling procedure

All patients scheduled for different elective surgical procedures in ATRH during the study period were included.

Data Collection And Measurment

Data was collected by using a Structured Questionnaire which contains general information and close end questionnaire. The response was to encircle or write in space provided. The data wascollected by selected $3^{\text {rd }}$ year anesthesia students in collaboration with principal investigators after orienting about the aim of study, how to collect data and reduce bias.

\subsection{Data Quality Assurance}

Data collectors and supervisors was trained for a half day on each items included in the study tools, objective, relevant of study, right of respondents, confidentiality of information obtained. During data collection, regular supervision and follow up was made. Investigator was check for completeness and consistency of data on daily basis. Once the data was collected and checked for completeness, consistency and accuracy, it was sorted, categorized and summarized.

\subsection{Data Analyzing and Processing}

The data was entered on epi info version 7 and was exported to SPSS version 20 computer program for analysis. Descriptive statistics was used to summarize data, tables and 
figures for display results.

\subsection{Operational Definitions Terms}

Surgery: is medical treatment in which a doctor cuts into someone's body in order to repair or remove damaged or diseased parts.

Elective surgery: - is a non-emergency surgery which is medically necessary, but.

which can be delayed for at least 24 hours.

Cancelation: - postponing or ignoring to do surgical procedure after planned and posted to do it.

\section{Ethical Consideration}

Ethical clearance and approval was obtained from ethical review committee, Anesthesia department, Arsi University. Informed written consent was secured from every study participant before the start of the interview after telling them about the objective of the study. The obtained data was only used for study purpose. Confidentiality and anonymity were ensured.

\section{Results}

Sociodemographic characteristics and cancellation of surgery

This study includes 112 elective patients with 100\% response rate. From this 60 were males and 52 were females $76(67.8 \%)$ Patients were done on the day of planned surgery and $36(32.2 \%)$ patients were canceled.

Freqeuncy of cancellation.

Over one third of patients were cancelled while over three fourth of planned surgery was done (Figure 1).

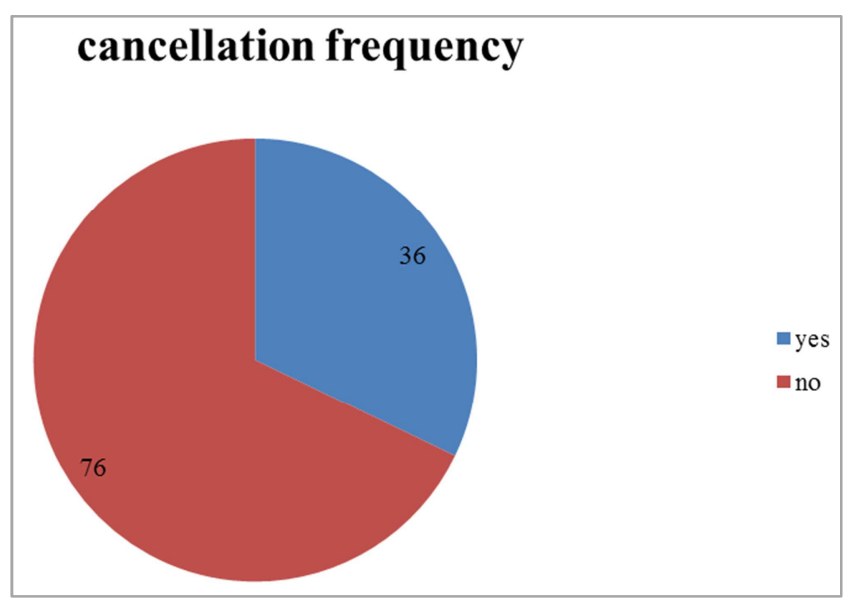

Figure 1. Frequency of surgical cancellation at Asella Teaching and Referral Hospital, 2018.

Magnitude of planned operation by department

From the total of 112 patients planned for surgery in our study period the highest number of cases were surgery which was $64(57.1 \%)$. Followed by gynecology and obstetrics $36(32.2 \%)$ and pediatrics the least $12(10.7 \%)$ of cases (Figure 2).

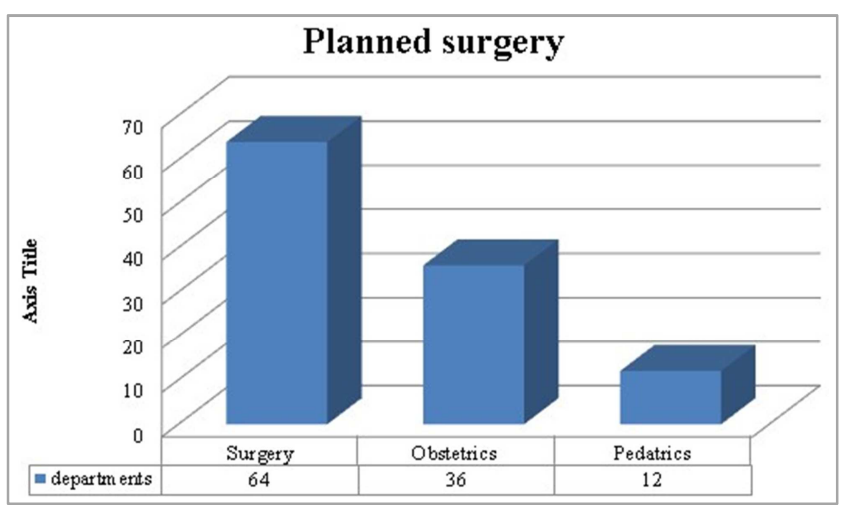

Figure 2. Magnitude of planned surgical at Asella Teaching and Referral Hospital, 2018.

Cancelation rate by age category

The age between 61 and 70 was the highest cancelation age group $13(36 \%)$ with the list under 10 years old and 11 20 years both had $1(2.5 \%)$ cancelation (Table 1$)$.

Table 1. Cancellation rate by age at Asella Teaching and Referral Hospital, 2018 .

\begin{tabular}{llllll}
\hline \multirow{2}{*}{ Count } & \multicolumn{2}{l}{ Age category } & \multirow{2}{*}{ Total } \\
\cline { 2 - 5 } & $\mathbf{1 ( \mathbf { 1 - 2 0 } )}$ & $\mathbf{( 2 1 - 4 0 )}$ & $\mathbf{( 4 1 - 6 0 )}$ & $\mathbf{( 6 1 - 8 0 )}$ & \\
\hline Canceled & 2 & 5 & 13 & 16 & 36 \\
Done & 9 & 13 & 24 & 30 & 76 \\
Total & 11 & 19 & 38 & 48 & 112 \\
\hline
\end{tabular}

Reasons of cancelation

From the total of 36 cases administration related cause of cancelation was the most common $17(47.2 \%)$ and medical problems were the second common with $9(25 \%)$ in the other hand no patient is canceled with anesthesia related causes. Nine patients were canceled by medical illness (8\%) from total 112 cases which hypertension was the highest according to the study (Table 2).

Table 2. Cancellation reason at Asella Teaching and Referral Hospital, 2018

\begin{tabular}{lll}
\hline Cause of cancelation & Frequency & Percent \\
\hline medical reasons & 9 & 25 \\
inadequate preparation of patient & 7 & 16.6 \\
patient related & 2 & 8.3 \\
administration related & 17 & 47.2 \\
Surgery/related anesthesia related & 1 & 2.7 \\
Total & 36 & 100 \\
\hline
\end{tabular}

Inadequate preparation as Cancellation

Highest frequent reason for inadequate patient preparation was blood is not available at the right time 4 (66.7\%) and investigation was incomplete was the second reasons, 2 $(33.3 \%)$ (Table 3$)$.

Table 3. Inadeqaute preparation as Cancellation at Asella Teaching and Referral Hospital, 2018.

\begin{tabular}{lll}
\hline Inadequate preparation & Frequency & Percent \\
\hline Blood is not prepared & 5 & 66.6 \\
Investigation not done & 2 & 33.3 \\
Total & 7 & 100 \\
\hline
\end{tabular}


Patient related reasons as Cancellation

From patient related causes of cancelation blood was not prepared was the most common cause for cancelation which is $7(71.4 \%)$ (Table 4$)$.

Table 4. Patient realted factors as Cancellation at Asella Teaching and Referral Hospital, 2018.

\begin{tabular}{lll}
\hline Patient related reasons & Frequency & Percent \\
\hline Patient is not fasting & 1 & 50 \\
Patient refusal & 1 & 50 \\
Total & 2 & 100 \\
\hline
\end{tabular}

Administrative related reasons

From total of 17 cases which canceled due to administrative related causes 7 (41.2) was canceled due to Overload schedule or and next to it due to no sterile cloth or autoclave was not done $6(23.5 \%)$ was canceled. From surgical related cause only $1(0.9 \%)$ case was canceled secondary to change in treatment plan and no case was canceled due to anesthesia related causes (Table 5).

Table 5. Administrative related factors as Cancellation at Asella Teaching and Referral Hospital, 2018.

\begin{tabular}{lll}
\hline Administration related & Frequency & Percent \\
\hline Overload schedule & 7 & 41.1 \\
No sterile cloth & 6 & 35.2 \\
Emergency priority & 2 & 11.8 \\
Previous case prolonged & 2 & 11.8 \\
Total & 17 & 100 \\
\hline
\end{tabular}

\section{Discussion}

Cancelation is one of the main problems which happen in the hospital specifically in the OR as it leads to patient scarification by paying a lot of money for bedding, transport and staying with their pain for long of time [7]. Cancelation also results in wastage of operation room time decrease work efficiency. [1]

The acceptable cancellation rate for elective surgical procedures is controversial but it has been said $5 \%$ to $10 \%$ by ASA [1]. The reported incidences for elective surgery cancellation vary widely among institutions from $10-40 \%$. [1, $3,5]$

Elective surgical case cancelation rate was $36(32.2 \%)$ in this research from 112 elective patient who were planned to do durig our study period which is higher when compared to study done in Jimma University $(23 \%)$. [3, 9]

When we see research done out of our country, research done in Saudi Arabia prevalence of cancelation at the day of surgery was $23.3 \%$. (15) It is $6.5 \%$ in research done in Spanish general hospital. [4]

The above couple of sentences' show us how much the problem is significant in our country especially in our hospital. This is because our setup has few operation room in proportion to patient flow compared with others. From 112 cases $60(53.5 \%)$ were male and $52(46.4 \%)$ were female. Male to Female ratio was 1.2:1 Cancelation rate in male was $36.6 \%$ and $27 \%$ in female. when we see in previous study which was done in Addis Ababa University the cancelation rate was high in male $37.2 \%$ than female which is $28.3 \%$. [3] which is also true for our hospital In this research the prevalence of cancelation was high in department of surgery which had poor scheduling method when compared with other departments.

Age from 41 to 60 was with high number of case which is planned for elective operation $38(32.8 \%)$ of case and higher number of case canceled is in the age group between 61 and 8016 , highest group of cancelation was 1 to 10 years old in TAH and in a research done in Spain but in Sudan the cancelation rate was higher in age 61-70 years old $31.1 \%$ followed by 51-60 years old group $25.4 \%$. AAU. [3]

In our study period from total 36 canceled cases surgery department has large number of cancelation 19, gynecology 13 and least pediatrics with 4 cases canceled. In most of the research done before orthopedics was a department with large number of cancelation 38 (30.4\%) in TAH 33.9\% in Saudi Arabia.

As a whole Patient related reasons for cancellation were 9 (7.8\%) which was $27(21.6 \%)$ in TAH 51\% (12) UK Glam organ hospital, in Finland $72.4 \%$ (11), in Saudi Arabia (32\%) and $10 \%(13)$ in china $(1,3,9)$. Those researches show that increased number of patient related cancelation is because the patient is shifted to the other hospitals [8]. Because there are no more choices in our country patient related cancelation is lower than the others.

Most cases were canceled by anesthetists $12(30 \%)$ patient $10(25 \%)$ the second, anesthetists were responsible for cancellation of $8 \%$ of total cancellations done on the day of surgery in similar research done in khan hospital [11].

If we compare cancelation rate of our hospital we may get it very poor and show us there is long journey in front to reach at the their level for example cancelation rate is more than $32.2 \%$ in our set up as we said before but in New South Wales, Australia, cancellations on the day of surgery was less than $2 \%$ and cancellation due to medical reasons was set at less than $1 \%$. [12] As it is described in other researches in this research also we gate most of the on day cancellations were due to potentially avoidable reasons As overload schedule is common cause of cancelation in our study estimating the operating time is also a very important factor to be kept in mind while preparing the OR schedule list for the next working day. In addition to the previously listed problems secondary to cancelation patient who canceled after they were NPO for couples of hours develop hypoglycemia and feels discomfort which affects the patients' attitude toward surgery.

Number of patients who canceled after they become NPO is $30(75 \%)$ in this study it is $40 \%$ in research done in Beijing hospital China. This is due to there is no clear job distribution in our hospital that means more than 3 persons may order the patient to be NPO or not. A possible solution could be to give written Instructions instead of verbal [11].

There is a need to discuss planning and risk of anesthesia with the patient and surgeon during preoperative evaluation to prevent day of surgery cancellation. The logistics of implementing this needs 
further discussion with the surgical department. Sometimes the cancellation occurred due to a difference of opinion between the anesthetist evaluating the patient and the anesthetist assigned for the surgery. It would be ideal if the same anesthetist who performed the preoperative assessment also conducted the anesthesia [13].

No patient was canceled for referral reason in our study period of time two patients were canceled by lack of postoperative ICU bed for better management in AAU, in South Africa no patient was canceled because of ICU bed shortage [21]. This is due to referral of patient before they planned to have a surgery this may be positive side in ATRH because it decreases the cost of the patient and increase their satisfaction by decreasing cancelation of cases. 9 (7.8\%) of the cases were canceled due to medical reasons in this study and the reasons for medical cancellations were reviewed, and the probable ones were inadvertently missing out on the abnormal results of the investigations done a day earlier, due to excess workload. Cancellations due to medical problems are especially upsetting for patients and should be avoided by devising appropriate protocols [11].

Large difference in rate of cancellation is seen depending on whether the study is retrospective or prospective. Pollard showed a $6.6 \%$ cancellation rate in a retrospective study and a cancellation rate of $13 \%$ in a prospective study which was twice that seen in the retrospective study [11]. So we should have kept in mind this while doing or interpreting this type researches.

\section{Conclusion}

Cancelation of elective surgical patient was high during our study period of time. Most of the reasons were administration related and shortage of time, medical reasons and inadequate patient preparation. Most of the reasons of cancellations of elective operations were avoidable. Some factors are beyond control of OR manager. Tackling each problem in the process, starting from initial booking to patient notification, will lead to quality improvement. The reasons of cancellation are inter-related.

\section{Limitations}

It was done with very small sample size due to time limitation.

Cases were not canceled according to the international criteria to cancel that means most of the case compromised due to our poor setup.

The research is done only in one season which makes impossible to know seasonal difference.

\section{Recommendation}

Patient preoperative evaluation should be before a weak from the day of surgery as an international standard that will help us to prepare the patient well. And preoperative evaluation and patient reassurance should be done as a team approach to have a common understanding among all professionals who involve in the operation. There should be good data handling and the hospital should plan according to the monthly report.

A long-term planning should be aimed at increasing operation theatre spaces equipped with enough facilities and manpower. It was known most of the reasons for cancellation were avoidable and can be prevented by different methods. Cancelation can be minimized if the patient with medical problems are detected early and referred for anesthetic evaluation as soon as scheduled for operation. We would like to recommend the implementation of control charts for monitoring cancellation of operations. Case cancellations can be reduced by improving early preoperative assessment, increasing patient's involvement, requesting patient information on inter-current illnesses between preadmission and surgery, better interdepartmental coordination and defining more clearly the reasons for theatre overruns in any future prospective study.

\section{Funding}

Arsi University.

\section{Competing Interests}

We declared that we had no competing interests.

\section{Authors' Contributions}

AT has contributed to conception, design of the study, data supervision, data entry clerk, data analyses, result interpretation, manuscript development and revision. GK and BT contributed data analyses, result interpretation and manuscript development. All authors read and approve the final manuscript.

\section{Acknowledgements}

We would like to thank all the staff in the of Arsi University for providing valuable information.

\section{References}

[1] Elective Surgery Patient Information. Government Of Western Australia. 2014, 1 (1): 1-2.

[2] Einar H. A New Pathway For Elective Surgery To Reduce Cancellation Rate. BMC Health Serviceresearch. 2012, Volume 12: 154 .

[3] Addis Shiferaw. Magnitude Of Case Cancellation And Associatedfactors Among Elective Surgical Cases In Tikur Anbesaspecialized Hospitalhospital, Addis Ababa, Ethiopia. 2016, 1 (1): 1-37.

[4] A. Gonzalez-Are'Valoetal. Causes For Cancellation Of Elective Surgical Procedures. Journalofthe Association Of Anesthetists Of Great Britain And Ireland. 2009, 1 (1): 487-493. 
[5] Arshadzafar, Tariq Saeed Mufti, Samson Griffin. CANCELLED ELECTIVE GENERAL SURGICAL OPERATIONS IN AYUB TEACHING HOSPITAL. 2007, 19 (3): 64-66.

[6] Martin Schuster, Christianneuman. The Effect Of Hospital Size And Surgical Services On Case Cancellation In Elective Surgery. International Anesthesia Research Society. 2011, 113 (3): $578-585$.

[7] Charisma. I. Why Is Surgery Cancelled? South Africa Journal Of Surgery. 2008, 3 (46): No Page Number.

[8] Ahmed A Elrahman, Aamir A Hamza, \& Mohayad A El-Haj. Cancellation Of Elective General Surgical Operations At The Day of Intended Surgery. Global Journal of HUMANSOCIAL SCIENCE: I Surgeries And Cardiovascular System. 2014, 14: 2249-4618.

[9] Haile M, Negadesalegn. Prospective Study Of Proportions And Causes Of Cancellation of Surgical Operations At Jimma University Teaching Hospital, Ethiopia. International Journal Of Anesthesiology \& Research. 2015, 3 (2): 87-90.
[10] Rakesh G, Anju, Poonam B, Nishkarshg, And Rakia. Reasons For Cancellation of Caseson The Day Of Surgery-A Prospective Study. Indianjoornal Of Anesthesia. 2009 Feb; 53 (1).

[11] Aziza Mohamed. Anesthetic Reasons For Cancellation Of Elective Surgical Inpatients On The Day Of Surgery In A Teaching Hospital. Aga Khan University Hospital. 2005, 55 (9): 374-378.

[12] Farhanulhuda. A Retrospective Analysis Of Reasons For Cancellation Of Elective Surgery In A Teaching Hospital. International Journal Of Scientific Study. 2014, 2 (2): 28-30.

[13] CH Chiu, A Lee, Phd, PT Chui. Cancellation Of Elective Operations On The Day Of Intended Surgery In A Hong Kong Hospital: Point Prevalence And Reasons. Hong Kong Med J. 2012, 18 (1): 5-10.

[14] Laisi J, Tomah, Kearney U. Surgery Cancelation On The Day of Surgery In Semenov. 3204-208. 